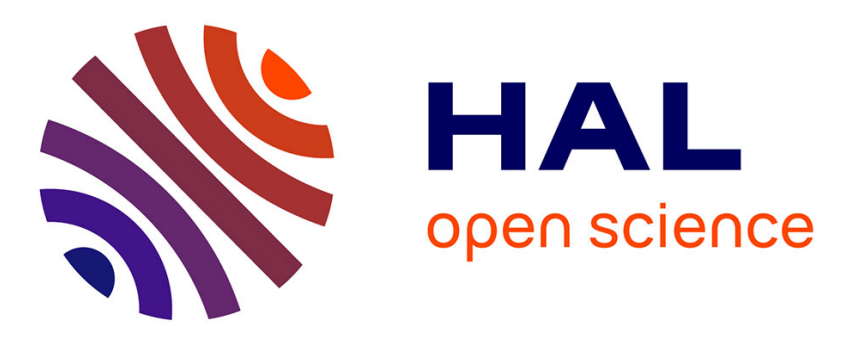

\title{
Moments of the total magnetization and conformal invariance in the finite two-dimensional Ising model
}

Theodore Burkhardt, Bernard Derrida

\section{To cite this version:}

Theodore Burkhardt, Bernard Derrida. Moments of the total magnetization and conformal invariance in the finite two-dimensional Ising model. Physical Review B, 1985, 32 (11), pp.7273-7279. 10.1103/PhysRevB.32.7273 . hal-03285920

\section{HAL Id: hal-03285920 \\ https://hal.science/hal-03285920}

Submitted on 19 Jul 2021

HAL is a multi-disciplinary open access archive for the deposit and dissemination of scientific research documents, whether they are published or not. The documents may come from teaching and research institutions in France or abroad, or from public or private research centers.
L'archive ouverte pluridisciplinaire $\mathbf{H A L}$, est destinée au dépôt et à la diffusion de documents scientifiques de niveau recherche, publiés ou non, émanant des établissements d'enseignement et de recherche français ou étrangers, des laboratoires publics ou privés. 


\title{
Moments of the total magnetization and conformal invariance in the finite two-dimensional Ising model
}

\author{
Theodore W. Burkhardt \\ Institut Laue-Langevin, Boite Postale 156X, F-38042 Grenoble Cedex, France \\ and Department of Physics, Temple University, ${ }^{*}$ Philadelphia, Pennsylvania 19122
}

Bernard Derrida

Service de Physique Théorique, Centre d'Etudes Nucléaires de Saclay, F-91191 Gif-sur-Yvette Cedex, France

(Received 21 June 1985)

\begin{abstract}
We consider Ising strips with width $N$ and periodic boundary conditions and Ising squares with edge length $N$ and special partially periodic boundaries. Assuming invariance of the spin correlations under conformal mappings of the infinite plane onto the strip and square, we determine the second and fourth moments of the total magnetization $M$ from the known bulk two- and four-spin correlation functions at criticality. For both geometries the predictions of conformal invariance for universal asymptotic forms involving the ratio $\left\langle M^{4}\right\rangle\left\langle M^{2}\right\rangle^{-2}$ as $N \rightarrow \infty$ are in excellent agreement with transfer-matrix results.
\end{abstract}

\section{INTRODUCTION}

The finite-size scaling technique known as phenomenological renormalization ${ }^{1,2}$ has proved to be an extremely reliable method for determining the critical properties of low-dimensional systems. In a typical application one is interested in quantities such as the critical coupling $K_{c}$ and the critical exponent $v$ of an infinite spin system in $d=2$ or 3 spatial dimensions. In phenomenological renormalization a more tractable geometry is considered. The system has length $L, L \rightarrow \infty$, in only one of the $d$ orthogonal directions and a finite width $N$ in the other $d-1$ directions. With a now standard procedure, estimates of $K_{c}$ and $v$ are obtained from the correlation lengths $\xi_{N}(K), \xi_{N^{\prime}}\left(K^{\prime}\right)$ for two different widths $N$ and $N^{\prime}$. The predictions generally converge rapidly toward the exact bulk values as $N$ and $N^{\prime}$ are increased.

Phenomenological renormalization studies also yield estimates of the universal amplitude

$$
A_{\xi}=\lim _{N \rightarrow \infty}\left[N^{-1} \xi_{N}\left(K_{c}\right)\right]
$$

that has been the subject of considerable attention. From numerical studies and exact calculations on a variety of two-dimensional models, it was observed ${ }^{3-6}$ that for strips of width $N$ and infinite length with periodic boundary conditions, the relation

$$
A_{\xi}=(\pi \eta)^{-1}
$$

is generally satisfied. Here, $\eta$ is the usual bulk critical exponent, i.e., in an infinite system at criticality the spinspin correlation function is given by

$$
G_{\infty}\left(z_{1}, z_{2}\right)=B\left|z_{1}-z_{2}\right|^{-\eta}
$$

for separations $\left|z_{1}-z_{2}\right|$ large in comparison with the lattice constant. We use complex notation $z=x+i y$ to specify points in the $x-y$ plane. Ordinary finite-size scal- ing arguments imply the universality of $A_{\xi}$ but not the relationship with $\eta$.

Recently, Cardy ${ }^{7}$ has pointed out that conformal invariance ${ }^{8-10}$ strongly restricts the form of correlations in confined geometries and, in particular, implies Eq. (1.2). Car$\mathrm{dy}^{11}$ has also used conformal invariance to determine the correlation functions and surface critical exponents of semi-infinite two-dimensional systems.

Binder ${ }^{12}$ has introduced a variant of phenomenological renormalization in which, for the hyperstrip geometry discussed above, the quantity

$$
U_{N}(K)=\lim _{L \rightarrow \infty}\left[L\left(1-\frac{1}{3}\left\langle M^{4}\right\rangle_{L, N}\left\langle M^{2}\right\rangle_{L, N}^{-2}\right)\right]
$$

rather than the correlation function $\xi_{N}(K)$ plays the central role. Here, $M$ denotes the total (extensive) magnetization. The factor $L$ and the subtraction in Eq. (1.4) ensure that $U_{N}(K)$ remains finite in the limit $L \rightarrow \infty$ of an infinitely long strip $(d=2)$ or bar $(d=3)$. For a hypercubic system no subtraction is necessary, and one may consider the quantity

$$
V_{N}(K)=\left\langle M^{4}\right\rangle_{N^{d}}\left\langle M^{2}\right\rangle_{N^{d}}^{-2} .
$$

It is generally more convenient to calculate $U_{N}(K)$ or $V_{N}(K)$ rather than $\xi_{N}(K)$ with Monte Carlo numerical methods and to consider hypercubes instead of hyperstrips. Some of the advantages of working with $U_{N}(K)$ in the site-percolation problem are discussed in Ref. 13, and numerical results for $d=2$ and 3 dimensions are reported.

According to the theory of finite-size scaling ${ }^{2,14} A_{\xi}$ and the quantities $A_{U}$ and $V^{*}$ defined by

$$
\begin{aligned}
A_{U} & =\lim _{N \rightarrow \infty}\left[N^{-1} U_{N}\left(K_{c}\right)\right], \\
V^{*} & =\lim _{N \rightarrow \infty}\left[V_{N}\left(K_{c}\right)\right],
\end{aligned}
$$

are universal in the same sense as the critical exponents. (Note, however, that the values of $A_{\xi}, A_{U}$, and $V^{*}$ do de- 
pend on boundary conditions. ${ }^{7}$ ) Estimates of $A_{U}$ and $V^{*}$ for the Ising model in various dimensions are given in Refs. 12, 13, and 15. Brézin and Zinn-Justin ${ }^{16}$ and Eisenriegler ${ }^{17}$ have applied field-theoretical methods to finitesize scaling. In Ref. 16 values of $A_{\xi}$ and $V^{*}$ are determined in $2+\epsilon$ and $4-\epsilon$ dimensions.

In this paper we study the finite-size scaling of $\left\langle M^{2}\right\rangle$ and $\left\langle M^{4}\right\rangle$ in Ising strips with periodic boundary conditions and in Ising squares having special partially periodic boundaries with an approach based on conformal invariance. The two- and four-spin correlations in the strip and square geometries are determined from the known bulk correlation functions by conformal mapping. The second and fourth moments of the total magnetization, which appear in Eqs. (1.4) and (1.5), are calculated by integrating the correlation functions. The values of $A_{U}$ and $V^{*}$ that we obtain are in excellent agreement with transfer-matrix results, which are also reported below. The agreement is evidence of the conformal invariance of both two- and four-spin correlations under coordinate transformations that change the geometry of the system fundamentally and, unlike the ordinary scaling transformation $\mathbf{r}^{\prime}=b^{-1} \mathbf{r}$, map infinite critical systems onto finite noncritical systems.

We note that Kleban et al. ${ }^{18}$ have recently calculated the structure factor $S(\mathbf{k})$ for several finite Ising systems with free boundaries with a similar approach based on conformal invariance.

The paper is organized as follows. In Sec. II we review the transformation properties of correlation functions under conformal mappings and discuss particular conformal mappings that map the infinite plane onto the strip and square. In Secs. III and IV, numerical values for $A_{U}$ and $V^{*}$ are derived on the basis of conformal invariance and compared with transfer-matrix results. Section V contains concluding remarks. The Monte Carlo integration procedure used to calculate moments of the total magnetization from correlation functions is described in the Appendix.

\section{CONFORMAL TRANSFORMATIONS AND CORRELATION FUNCTIONS}

A coordinate transformation $\mathbf{r} \rightarrow \mathbf{r}^{\prime}$ is conformal ${ }^{8,9}$ if it preserves angles, i.e., corresponds locally to a translation, a rotation, and a dilation. Two-dimensional conformal mappings can be represented in the form $z \rightarrow w$, where $w=u+i v$ is an arbitrary analytic function of the complex variable $z=x+i y$. In higher dimensions the conformal group is less rich, consisting of homogeneous translations, rotations, and dilations and special transformations $\mathbf{r}^{\prime}\left|\mathbf{r}^{\prime}\right|^{-2}=\mathbf{r}|\mathbf{r}|^{-2}+\mathbf{a}$, where $\mathbf{a}$ is an arbitrary constant vector, that map any hyperspherical surface onto another.

Consider a single-component spin system in the continuum limit, i.e., with an infinitesimal lattice constant. According to the conformal-invariance hypothesis, the $n$ spin correlation function transforms according to 7,11

$$
\begin{aligned}
G_{g^{\prime}}\left(\mathbf{r}_{1}^{\prime}, \ldots, \mathbf{r}_{n}^{\prime}\right)= & b\left(\mathbf{r}_{1}\right)^{(d-2+\eta) / 2} \ldots \\
& \times b\left(\mathbf{r}_{n}\right)^{(d-2+\eta) / 2} G_{g}\left(\mathbf{r}_{1}, \ldots, \mathbf{r}_{n}\right),
\end{aligned}
$$

under a conformal coordinate transformation. The subscripts $g$ and $g^{\prime}$ refer to the boundary geometry, which, in general, is modified by the conformal transformation. The quantity $b(\mathbf{r})$ specifies the local change in the length scale, i.e., $b(\mathbf{r})^{-d}=\left|\partial \mathbf{r}^{\prime} / \partial \mathbf{r}\right|$ is the Jacobian of the transformation. For spatially homogeneous $b$, Eq. (2.1) reduces to the ordinary transformation equation for an infinite critical system under the scale change $\mathbf{r}^{\prime}=b^{-1} \mathbf{r}$.

The correlation functions in Eqs. (2.1) are multiple derivatives of the free energy with respect to the original and renormalized local fields $h_{\mathrm{r}}$ and $h_{\mathrm{r}^{\prime}}^{\prime}$, respectively. Equation (2.1) follows from the assumption that these fields are related by a local renormalization transformation.

For the two-dimensional conformal mapping generated by the analytic function $w(z)$, the length-rescaling factor is given by $b(\mathbf{r})=\left|w^{\prime}(z)\right|^{-1}$, where the prime denotes a derivative with respect to $z$. Thus, in two dimensions Eq. (2.1) may be rewritten as 7,11

$$
\begin{aligned}
G_{g^{\prime}}\left(w_{1}, \ldots, w_{n}\right)= & \mid w^{\prime}\left(z_{1}\right) \cdots \\
& \times\left. w^{\prime}\left(z_{n}\right)\right|^{-\eta / 2} G_{g}\left(z_{1}, \ldots, z_{n}\right) .
\end{aligned}
$$

To determine correlations in the strip geometry from bulk correlations, we use the function ${ }^{7}$

$$
w(z)=\frac{N}{2 \pi} \ln z
$$

that maps the entire $z$ plane onto the strip $-\infty<u<\infty$, $0<v<N$, where $z=x+i y$ and $w=u+i v$. Some of the contours of constant $u$ and $v$ in the $z$ plane, corresponding to Eq. (2.3), are shown in Fig. 1(a). A full set of contours in the $z$ plane with separation $|d u|=|d v|=\delta a$ (infinitesimal constant) may be interpreted as a spin system on a locally square lattice with a nonuniform lattice constant

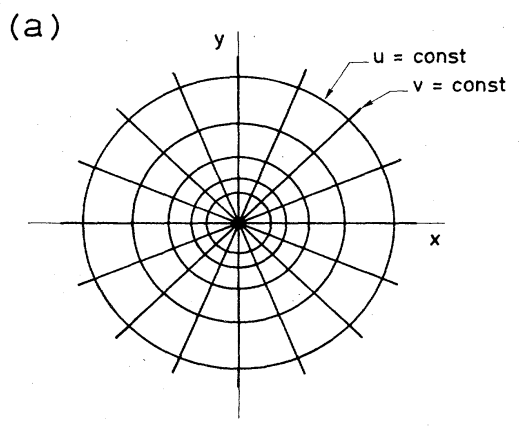

(b)

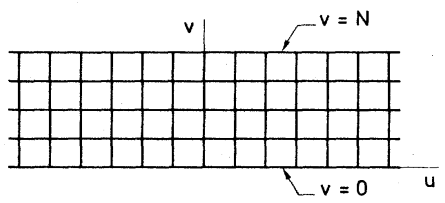

FIG. 1. (a) Contours of constant $u$ (circles) and of constant $v$ (radial lines) in the $z$ plane for the logarithmic mapping of Eq. (2.3). (b) Corresponding contours in the $w$ plane. Since the positive $x$ axis in (a) represents both $v=0$ and $v=N$, the infinite strip has periodic boundary conditions. 
$\left|w^{\prime}(z)\right|^{-1} \delta a$, as depicted schematically in Fig. 1(a). One can imagine generating this spin system by carrying out a spatially inhomogeneous renormalization with scale factor $b(z)=\left|w^{\prime}(z)\right|^{-1}$ on an infinite initial system in the $z$ plane with uniform lattice constant $\delta a$. If the initial system has isotropic critical couplings, the renormalized system does also. Thus, the renormalized system is topologically equivalent to a spin system on a strip of infinite length and finite width, with a uniform infinitesimal lattice constant and with periodic boundary conditions, as shown schematically in Fig. 1(b). To emphasize the periodicity, one may, of course, draw the strip on a cylindrical surface.

Following $\mathrm{Cardy},{ }^{7}$ we determine the pair correlation function $G_{N}\left(w_{1}, w_{2}\right)$ for the strip by inserting the bulk correlation function $G_{\infty}\left(z_{1}, z_{2}\right)$ of Eq. (1.3) and Eq. (2.3) into Eq. (2.2). A short calculation gives

$$
\begin{aligned}
G_{N}\left(w_{1}, w_{2}\right)=B\left\{\left(\frac{N}{2 \pi}\right)^{2}\right. & {\left[2 \cosh \left[\frac{2 \pi}{N}\left(u_{1}-u_{2}\right)\right]\right.} \\
& \left.\left.-2 \cos \left[\frac{2 \pi}{N}\left(v_{1}-v_{2}\right)\right]\right]\right\}^{-\eta / 2}
\end{aligned}
$$

The correlation function (2.4) is translationally invariant and periodic in $v_{1}-v_{2}$. For $\left|w_{1}-w_{2}\right|<<N$ it takes the same form as the bulk correlation function, as expected. For $\left|w_{1}-w_{2}\right| \gg N, G_{N}$ decays as $\exp \left(-\pi \eta \mid u_{1}\right.$ $\left.-u_{2} \mid / N\right)$, with a correlation length given by Eqs. (1.1) and (1.2). This is Cardy's derivation of Eq. (1.2).

Ordinary scaling completely dictates the form (1.3) of the bulk pair correlation function at a critical point. In the case of four- and more-spin correlations, neither ordinary scaling nor the stronger requirement of invariance under the small conformal group ${ }^{19}$ entirely determine the spatial dependence. Exact results ${ }^{20}$ are available for the bulk critical four-spin correlations of the two-dimensional Ising model in the continuum limit. The four-spin correlation function is given in terms of pair correlations by

$$
\begin{aligned}
G(1,2,3,4)=\frac{1}{\sqrt{2}}[ & {\left[\frac{G(1,2) G(2,3) G(3,4) G(4,1)}{G(1,3) G(2,4)}\right]^{2} } \\
& +(2 \leftrightarrow 3)+(3 \leftrightarrow 4)]^{1 / 2}
\end{aligned}
$$

in an obvious abbreviated notation. The pair correlation functions have the form (1.3), with $\eta=\frac{1}{4}$.

Equation (2.5) is established in Ref. 20 for the infinite two-dimensional Ising model at criticality. If both the two- and four-spin correlation functions transform according to Eq. (2.2), the structure of Eq. (2.5) is preserved by conformal mapping. Thus, we obtain an expression for the four-spin correlation function in an Ising strip of width $N$ by inserting Eq. (2.4) into Eq. (2.5).

To determine the two- and four-spin correlation functions in a finite rectangle, we utilize the SchwarzChristoffel mapping ${ }^{21}$

$$
w=C \int_{0}^{z} d t\left[\left(1-t^{2}\right)\left(1-k^{2} t^{2}\right)\right]^{-1 / 2}
$$

of the upper-half $z$ plane onto a rectangle. The constants $C$ and $k$ are fixed by the requirements that the points $z= \pm 1$ and $z= \pm k^{-1}$, with $k^{-1}>1$, on the $x$ axis map onto the corners $w= \pm \frac{1}{2} a$ and $w= \pm \frac{1}{2} a+\frac{1}{2} i b$ of the rectangle, respectively, where $a$ and $b$ are real and positive.

The integral (2.6) is an elliptic integral of the first kind $^{22}$ and cannot be evaluated in closed form in terms of more elementary functions. By making simple changes of variables, it is easy to show that

$$
\begin{aligned}
& \frac{a}{2}=C F(k), \\
& \left.\frac{b}{2}=C F\left(1-k^{2}\right)^{1 / 2}\right),
\end{aligned}
$$

where $F(k)$ denotes the complete elliptical integral of the first kind, ${ }^{22}$ defined by

$$
F(k)=\int_{0}^{\pi / 2} d \theta\left(1-k^{2} \sin ^{2} \theta\right)^{-1 / 2} .
$$

Figure 2(a) shows a few of the contours of constant $u$ and $v$ in the upper-half $z$ plane for the mapping (2.6) with $k=2^{-1 / 2}$, which implies $a=b$. These contours may be continued into the lower-half $z$ plane by reflection. For

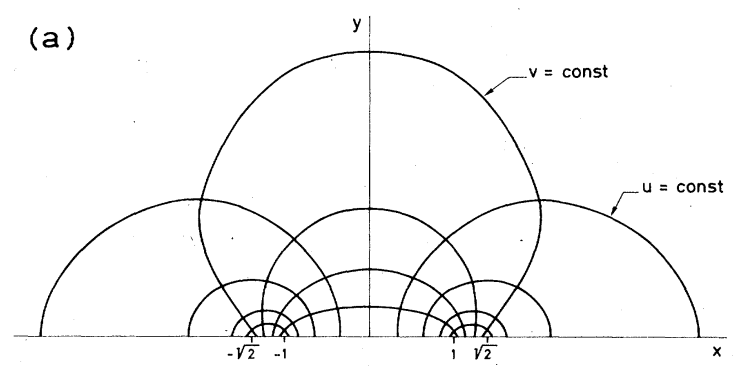

(b)
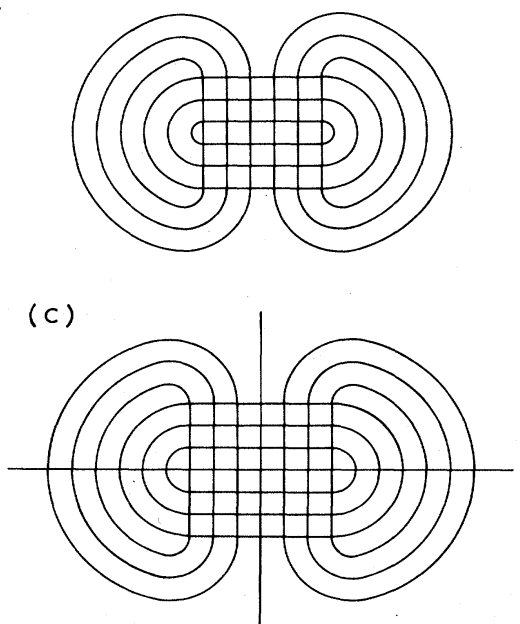

FIG. 2. (a) Contours of constant $u$ and $v$ in the upper half $z$ plane for the mapping of Eq. (2.6) with $k=2^{-1 / 2}$. (b) Corresponding contours defining a rectangle with partially periodic boundary conditions in the $w$ plane. An even number of constant- $v$ contours is shown. (c) Corresponding contours defining a rectangle with partially periodic boundary conditions in the $w$ plane. An odd number of constant- $v$ contours is shown. 
any $k^{-1}>1$, the full set of continued contours maps the entire $z$ plane onto a rectangle with corners at $w= \pm \frac{1}{2} a \pm \frac{1}{2} i b$. For $k=2^{-1 / 2}$ a square is obtained. As discussed above, we interpret the contours in the $z$ plane as a spin system with a nonuniform infinitesimal lattice constant but uniform interactions generated by the inhomogeneous renormalization of an infinite homogeneous system at the critical point. The system of spins with nonuniform lattice constant is topologically equivalent to a spin system with uniform interactions and uniform infinitesimal lattice constant defined on a finite rectangle. The rectangular system is shown schematically in Figs. 2(b) and 2(c) for even and odd numbers of constant- $v$ contours and with a lattice constant that is finite rather than infinitesimal. Note the unusual partially periodic boundary conditions. Every lattice site has coordination number 4 , but because of the boundary conditions the system is not translationally invariant.

\section{RESULTS FOR ISING STRIPS}

In this section the finite-size scaling behavior of $\left\langle M^{2}\right\rangle$ and $\left\langle M^{4}\right\rangle$ implied by conformal invariance is derived for Ising strips with periodic boundary conditions. The value of the amplitude $A_{U}$ of Eqs. (1.4) and (1.6a) is determined and compared with transfer-matrix results. We begin by defining

$$
\begin{aligned}
& m_{2}(N)=\lim _{L \rightarrow \infty}\left[(L N)^{-1}\left\langle M^{2}\right\rangle_{L, N}\right], \\
& m_{4}(N)=\lim _{L \rightarrow \infty}\left[(L N)^{-1}\left(\left\langle M^{4}\right\rangle_{L, N}-3\left\langle M^{2}\right\rangle_{L, N}^{2}\right)\right] .
\end{aligned}
$$

In the continuum limit the two- and four-spin correlation functions in the strip are given by Eqs. (2.4) and (2.5), as discussed above. In terms of these correlation functions

$$
\begin{aligned}
& m_{2}(N)=\rho^{2} \int_{-\infty}^{\infty} d u_{1} \int_{0}^{N} d v_{1} G_{N}\left(w_{1}, 0\right) \\
& m_{4}(N)=\rho^{4} \int_{-\infty}^{\infty} d u_{1} \int_{0}^{N} d v_{1} \cdots \int_{-\infty}^{\infty} d u_{3} \int_{0}^{N} d v_{3}\left[G_{N}\left(w_{1}, w_{2}, w_{3}, 0\right)-G_{N}\left(w_{1}, w_{2}\right) G_{N}\left(w_{3}, 0\right)\right. \\
& \left.\quad-G_{N}\left(w_{1}, w_{3}\right) G_{N}\left(w_{2}, 0\right)-G_{N}\left(w_{2}, w_{3}\right) G_{N}\left(w_{1}, 0\right)\right]
\end{aligned}
$$

Equations (3.2) utilize the translational invariance of the correlation functions. The quantity $\rho$ is the areal density of the spins. The integral defined by Eq. (3.2b) is infinite without the terms in the integrand preceeded by minus signs. Their subtraction corresponds to the subtraction in Eq. (1.4).

Making the change of integration variables $u_{i}^{\prime}=2 \pi u_{i} / N, v_{i}^{\prime}=2 \pi v_{i} / N$ in Eqs. (3.2), and using Eqs. (2.4), (2.5), and (3.2), one obtains

$$
\begin{aligned}
& m_{2}(N)=\left(\frac{N}{2 \pi}\right)^{7 / 4} m_{2}(2 \pi), \\
& m_{4}(N)=\left(\frac{N}{2 \pi}\right)^{11 / 2} m_{4}(2 \pi) .
\end{aligned}
$$

The integrals $m_{2}(2 \pi)$ and $m_{4}(2 \pi)$ were evaluated with a Monte Carlo numerical procedure described in the Appendix. Inserting the results into Eqs. (3.3) gives

$$
\begin{aligned}
& m_{2}(N)=(4.03641 \pm 0.00002) N^{7 / 4} \rho^{2} B, \\
& m_{4}(N)=-(120.2613 \pm 0.0009) N^{11 / 2} \rho^{4} B^{2}
\end{aligned}
$$

We see that conformal invariance completely determines the moments $m_{2}(N)$ and $m_{4}(N)$ defined by Eqs. (3.1) in terms of $N, \rho$ (spin density), and $B$ (amplitude of the bulk correlation function). The $N$ dependence of Eqs. (3.4) is consistent with ordinary dimensional analysis $^{23}$ and with finite-size scaling, ${ }^{2,14}$ which predicts $m_{2}(N)=\partial^{2} f / \partial h^{2} \sim N^{\gamma / v}$ and $m_{4}(N)=\partial^{4} f / \partial h^{4}$ $\sim N^{2 \gamma / v+d}$, as follows from the scaling form ${ }^{2,14}$

$$
f(t, h, N)=N^{-d} \psi\left(N^{y_{t}} t, N^{y_{h}} h\right)
$$

of the free energy per spin.
Equations (1.4), (1.6a), (3.1), and (3.3) imply

$$
A_{U}=-\frac{1}{3} \lim _{N \rightarrow \infty}\left[\frac{m_{4}(N)}{\left[N m_{2}(N)\right]^{2}}\right]=-\frac{1}{3} \frac{m_{4}(2 \pi)}{\left[2 \pi m_{2}(2 \pi)\right]^{2}} \text {. }
$$

The Monte Carlo calculation yields

$$
A_{U}=2.46044 \pm 0.00002 \text {. }
$$

Note that the universal amplitude $A_{U}$ is independent of the nonuniversal quantities $\rho$ and $B$, as is clear from Eqs. (3.4) and (3.6).

TABLE I. Transfer-matrix results for Ising strips with periodic boundary conditions and widths of $N=2,3, \ldots, 12$ lattice constants. In the limit $N \rightarrow \infty$ the quantity $U_{N}\left(K_{c}\right) / N$ approaches the universal amplitude $A_{U}$. The entry for $N=\infty$ is the value of $A_{U}$ predicted by conformal invariance.

\begin{tabular}{rl}
\hline$N$ & \multicolumn{1}{c}{$U_{N}\left(K_{c}\right) / N$} \\
\hline 2 & 2.24511 \\
3 & 2.34026 \\
4 & 2.39547 \\
5 & 2.42213 \\
6 & 2.43554 \\
7 & 2.44296 \\
8 & 2.44748 \\
9 & 2.45044 \\
10 & 2.45250 \\
11 & 2.45398 \\
12 & 2.45508 \\
$\infty$ & $2.46044 \pm 0.00002$ \\
\hline \hline
\end{tabular}


To check the prediction (3.7) of conformal invariance for the quantity $A_{U}$, we have calculated $U_{N}\left(K_{c}\right) / N$ for the Ising model on strips of a square lattice with widths $N=2,3, \ldots, 12$ lattice constants, using the transfermatrix approach of Saleur and Derrida. ${ }^{13}$ From Eq. (1.6a) one sees that $U_{N}\left(K_{c}\right) / N$ tends to $A_{U}$ in the large- $N$ limit. The transfer-matrix results and the prediction of conformal invariance are compared in Table I and Fig. 3. The agreement is excellent.

\section{RESULTS FOR ISING SQUARES}

In this section we derive the finite-size scaling behavior of $\left\langle M^{2}\right\rangle$ and $\left\langle M^{4}\right\rangle$ implied by conformal invariance for Ising squares with the partially periodic boundary conditions of Fig. 2. The numerical value of the universal quantity $V^{*}$ of Eqs. (1.5) and (1.6b) is determined and compared with transfer-matrix results.

For a spin continuum defined on the square with corners at $w= \pm \frac{1}{2} N \pm \frac{1}{2} i N,\left\langle M^{2}\right\rangle_{N, N}$ is related to the two-spin correlation function $G_{N, N}\left(w_{1}, w_{2}\right)$ by

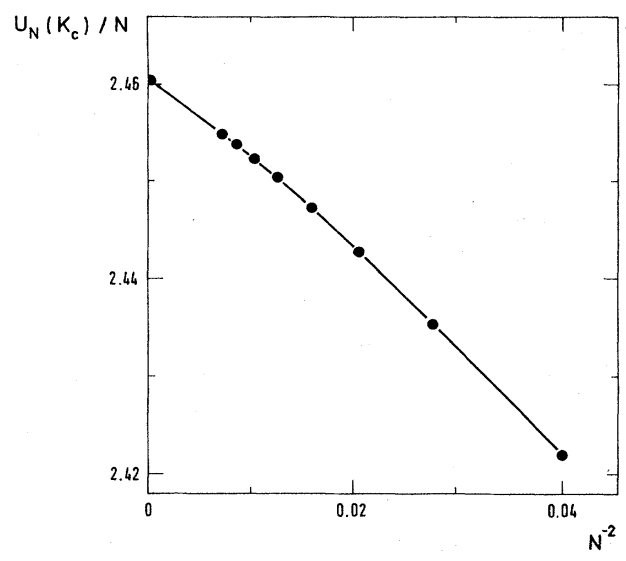

FIG. 3, $U_{N}\left(K_{c}\right) / N$ as a function of $N^{-2}$ for Ising strips with periodic boundary conditions and widths of $N=5,6, \ldots, 12$ lattice constants, calculated with the transfer-matrix method. The point on the vertical axis corresponding to $N=\infty$ is the prediction (3.7) of conformal invariance for $\boldsymbol{A}_{\boldsymbol{u}}$.

$$
\left\langle M^{2}\right\rangle_{N, N}=\rho^{2} \int_{-N / 2}^{N / 2} d u_{1} \int_{-N / 2}^{N / 2} d v_{1} \int_{-N / 2}^{N / 2} d u_{2} \int_{-N / 2}^{N / 2} d v_{2} G_{N, N}\left(w_{1}, w_{2}\right)
$$

where, as in the preceding section, $\rho$ is the areal density of the spins. An analogous formula relates $\left\langle M^{4}\right\rangle_{N, N}$ to the four-spin correlation function. Expressing the correlation functions for the finite square in terms of bulk correlation functions with the help of Eq. (2.2) and converting the $u, v$ integrations to $x, y$ integrations, one obtains

$$
\begin{aligned}
& \left\langle M^{2}\right\rangle_{N, N}=\rho^{2} \int_{-\infty}^{\infty} d x_{1} \int_{-\infty}^{\infty} d y_{1} \int_{-\infty}^{\infty} d x_{2} \int_{-\infty}^{\infty} d y_{2}\left|w^{\prime}\left(z_{1}\right) w^{\prime}\left(z_{2}\right)\right|^{2-\eta / 2} G_{\infty}\left(z_{1}, z_{2}\right), \\
& \left\langle M^{4}\right\rangle_{N, N}=\rho^{4} \int_{-\infty}^{\infty} d x_{1} \int_{-\infty}^{\infty} d y_{1} \cdots \int_{-\infty}^{\infty} d x_{4} \int_{-\infty}^{\infty} d y_{4}\left|w^{\prime}\left(z_{1}\right) \cdots w^{\prime}\left(z_{4}\right)\right|^{2-\eta / 2} G_{\infty}\left(z_{1}, \ldots, z_{4}\right) .
\end{aligned}
$$

The bulk correlation functions are given by Eqs. (1.3) and (2.5) with $\eta=\frac{1}{4}$, and

$$
\left|w^{\prime}(z)\right|=C\left|\left(1-z^{2}\right)\left(1-\frac{1}{2} z^{2}\right)\right|^{-1 / 2}
$$

as follows from Eq. (2.6) with $k=2^{-1 / 2}$, corresponding to a square with edge length $N$. Equation (2.7) implies $^{22}$ $\frac{1}{2} N=C F\left(2^{-1 / 2}\right)=1.85407 C$.

We have evaluated the integrals (4.2) with the Monte Carlo procedure described in the Appendix and find

$$
\begin{aligned}
& \left\langle M^{2}\right\rangle_{N, N}=(1.243 \pm 0.002) N^{15 / 4} \rho^{2} B, \\
& \left\langle M^{4}\right\rangle_{N, N}=(2.06 \pm 0.03) N^{15 / 2} \rho^{4} B^{2} .
\end{aligned}
$$

Again the $N$ dependence is consistent with ordinary dimensional analysis ${ }^{23}$ and with finite-size scaling, which implies [see Eq. (3.5)]

$$
\left\langle M^{2}\right\rangle_{N, N}=N^{d} \partial^{2} f / \partial h^{2} \sim N^{\gamma / v+d}
$$

and

$$
\left\langle M^{4}\right\rangle_{N, N}-3\left\langle M^{2}\right\rangle_{N, N}^{2}=N^{d} \partial^{4} f / \partial h^{4} \sim N^{2(\gamma / v+d)}
$$

for a hypercube with edges of length $N$. Conformal invariance not only predicts the $N$ dependence of the moments of the magnetization in Eqs. (4.4), but also determines the proportionality constants.

In the ratio $\left\langle M^{4}\right\rangle_{N, N}\left\langle M^{2}\right\rangle_{N, N}^{-2}$ the factors of $N$ and the nonuniversal quantities $\rho$ and $B$ cancel. For the universal limit $V^{*}$ of Eq. (1.6b), the Monte Carlo result is

$$
V^{*}=1.33 \pm 0.02 \text {. }
$$

To check the prediction of conformal invariance for $V^{*}$, we have calculated the quantity $V_{N}\left(K_{c}\right)$ of Eq. (1.5) for Ising squares with edges of length $N=2,4, \ldots, 14$ lattice constants, using the transfer-matrix approach of Saleur and Derrida. ${ }^{13}$ The partially periodic boundary conditions for even $N$ indicated in Fig. 2(b) were imposed. (Large- $N$ transfer-matrix calculations with the odd- $N$ boundary condition of Fig. 2(c) are considerably more difficult.) The results are given in Table II. The $V_{N}\left(K_{c}\right)$ with partially periodic boundaries and $N$ even approach a limiting value entirely consistent with the value of $V^{*}$ predicted by conformal invariance. A sequence of $V_{N}\left(K_{c}\right)$ for Ising squares with ordinary completely periodic boundary conditions is also shown in Table II for comparison. This sequence, which is consistent with the Monte Carlo results of Blöte and Bruce, ${ }^{15}$ extrapolates to a value of $V^{*}$ about $13 \%$ smaller than for partially periodic boundaries.

\section{CONCLUDING REMARKS}

The success of the conformal-invariance approach in predicting $A_{U}$ and $V^{*}$ for the Ising model provides further evidence that in the continuum limit, finite-size scaling behavior is fixed by bulk critical behavior when the 
TABLE II. Transfer-matrix results for $V_{N}\left(K_{c}\right)$ in Ising squares. The sequence marked partially periodic was calculated with the even- $N$ boundary conditions of Fig. 2(b). The entry for $N=\infty$ is the limiting value $V^{*}$ predicted by conformal invariance for this boundary condition. A sequence of $V_{N}\left(K_{c}\right)$ with completely periodic boundaries is shown for comparison.

\begin{tabular}{rcc}
\hline \hline$N$ & $\begin{array}{c}\text { Partially } \\
\text { periodic }\end{array}$ & $\begin{array}{c}\text { Completely } \\
\text { periodic }\end{array}$ \\
\hline 2 & 1.23122 & 1.11929 \\
3 & & 1.13956 \\
4 & 1.31183 & 1.14840 \\
5 & & 1.15357 \\
6 & 1.32914 & 1.15693 \\
7 & & 1.15923 \\
8 & 1.33635 & 1.16086 \\
9 & & 1.16207 \\
10 & 1.34003 & 1.16298 \\
11 & & 1.16369 \\
12 & 1.34217 & 1.16425 \\
13 & & 1.16470 \\
14 & 1.34352 & 1.16507 \\
$\infty$ & $1.33 \pm 0.02$ & \\
\hline
\end{tabular}

finite and infinite systems are related by a conformal mapping. Several model calculations ${ }^{3-6,24,25}$ have confirmed Eq. (2.2) for two-spin correlations with mappings of the plane or half plane onto an infinite strip or wedge. We have considered four-spin correlations as well and the more extreme mapping of the entire $z$ plane onto a square.

As discussed above, the mapping (2.6) of the $z$ plane onto a rectangle generates partially periodic boundary conditions, as shown in Fig. 2. It does not appear possible to derive correlations in a rectangle with completely periodic boundary conditions from bulk correlations using the approach of this paper.

Pair correlations in several finite Ising systems with free boundary conditions have been calculated by Kleban et al. ${ }^{18}$ by conformal mapping of the results for the half plane. $^{11}$ The four-point correlation function of the twodimensional semi-infinite Ising model can, in principle, be determined by the conformal-invariance method used by Cardy ${ }^{11}$ to obtain the pair correlation function. Thus it appears possible to calculate $A_{U}$ and $V^{*}$ for the Ising model (and other two-dimensional models) with free boundary conditions with the approach used here and in Ref. 18.

Finally, we note that the spatial dependence of the bulk four-point function at criticality is not uniquely fixed ${ }^{19}$ by the critical exponent $\eta$, unlike the spatial dependence (1.3) of the two-point function. For this reason there do not seem to exist simple general relations between $A_{U}$ and $\eta$ and $V^{*}$ and $\eta$ analogous to $A_{\xi}=(\pi \eta)^{-1}$.

\section{APPENDIX}

We briefly outline the Monte Carlo procedure used to calculate $\left\langle M^{2}\right\rangle$ and $\left\langle M^{4}\right\rangle$ from the two- and four-spin correlation functions for the strip and square geometries. For Ising strips the normalized second moment $m_{2}(N)$ of Eq. (3.1a) is given by

$$
\begin{aligned}
& m_{2}(N)=\left[\frac{N}{2 \pi}\right]^{7 / 4} \rho^{2} B I_{2}, \\
& I_{2}=\int_{-\infty}^{\infty} d u \int_{0}^{2 \pi} d v(2 \cosh u-2 \cos v)^{-1 / 8},
\end{aligned}
$$

according to Eqs. (2.4), (3.2a), and (3.3a). With the substitution $u=\ln s$, the domain of integration becomes finite, and $I_{2}$ takes the form

$$
I_{2}=2 \int_{0}^{1} d s \int_{0}^{2 \pi} d v s^{-7 / 8}\left(s^{2}-2 s \cos v+1\right)^{-1 / 8} \text {. }
$$

To weaken the $s^{-7 / 8}$ singularity, so that the integrand becomes square integrable, ${ }^{26}$ we make the additional substitution $s=t^{8}$ and obtain

$$
I_{2}=16 \int_{0}^{1} d t \int_{0}^{2 \pi} d v\left(t^{16}-2 t^{8} \cos v+1\right)^{-1 / 8} .
$$

In our Monte Carlo procedure this integral was approximated by the sum

$$
I_{2}=32 \pi \mathscr{N}^{-1} \sum_{i=1}^{\mathscr{N}}\left(t_{i}^{16}-2 t_{i}^{8} \cos v_{i}+1\right)^{-1 / 8},
$$

with the $t_{i}$ and $v_{i}$ chosen at random in the intervals $1<t_{i}<0,0<v_{i}<2 \pi$, respectively. The fourth moment $m_{4}(N)$ defined by Eqs. (3.1b) and (3.2b) was evaluated in a completely analogous fashion, with the same substitutions for the original integration variables. The results quoted in Eqs. (3.4) and (3.7) represent the average and standard error of eight determinations of each integral with $5 \times 10^{6}$ Monte Carlo steps per determination.

For Ising squares $\left\langle M^{2}\right\rangle_{N, N}$ can be written in the form

$$
\begin{aligned}
& \left\langle M^{2}\right\rangle_{N, N}=\rho^{2} B C^{15 / 4} J_{2} \\
& J_{2}=\int_{-\infty}^{\infty} d x_{1} \int_{-\infty}^{\infty} d y_{1} \int_{-\infty}^{\infty} d x_{2} \int_{-\infty}^{\infty} d y_{2}\left|\left(1-z_{1}^{2}\right)\left(1-\frac{1}{2} z_{1}^{2}\right)\left(1-z_{2}^{2}\right)\left(1-\frac{1}{2} z_{2}^{2}\right)\right|^{-15 / 16}\left|z_{1}-z_{2}\right|^{-1 / 4}
\end{aligned}
$$

with the help of Eqs. (4.2a) and (4.3). To put the integral in a more convenient form for numerical integration, we make the substitutions

$$
\begin{aligned}
& z_{1}^{2}=\frac{S_{1}}{1-S_{1}} \exp \left(i \phi_{1}\right), \\
& z_{2}^{2}=\frac{S_{2}}{1-S_{2}} \exp \left(i \phi_{2}\right),
\end{aligned}
$$

and obtain

$$
J_{2}=\int_{0}^{1} d S_{1} \int_{0}^{4 \pi} d \phi_{1} \int_{0}^{1} d S_{2} \int_{0}^{4 \pi} d \phi_{2} F\left(S_{1}, \phi_{1}, S_{2}, \phi_{2}\right) .
$$

We omit the explicit form of the integrand $F\left(S_{1}, \phi_{1}, S_{2}, \phi_{2}\right)$, which is long but simple to derive. The 
integrand has multiple singularities but is square integrable. ${ }^{26}$ In our Monte Carlo evaluation $J_{2}$ was approximated by

$$
J_{2}=(4 \pi)^{2} \mathscr{N}^{-1} \sum_{i=1}^{\mathscr{N}} F\left(S_{1 i} \phi_{1 i}, S_{2 i}, \phi_{2 i}\right)
$$

with $S_{1 i}, \ldots, \phi_{2 i}$ chosen randomly in the intervals $0<S_{1 i}<1, \ldots, 0<\phi_{2 i}<4 \pi$. The moment $\left\langle M^{4}\right\rangle_{N, N}$ of Eq. (4.2b) was evaluated similarly, with the same substitutions for the original integration variables. The results quoted in Eqs. (4.4) and (4.5) represent the average and standard error of 100 determinations of each integral with $10^{5}$ Monte Carlo steps per determination.
*Permanent address.

${ }^{1}$ M. P. Nightingale, J. Appl. Phys. 53, 7927 (1982).

${ }^{2}$ M. N. Barber, in Phase Transitions and Critical Phenomena, edited by C. Domb and J. L. Lebowitz (Academic, London, 1983), Vol. 8.

3J. L. Pichard and G. Sarma, J. Phys. C 14, L127 (1981); 14, L617 (1981).

4J. M. Luck, J. Phys. A 15, L169 (1982).

${ }^{5}$ B. Derrida and L. de Seze, J. Phys. (Paris) 43, 475 (1982).

${ }^{6}$ M. P. Nightingale and H. Blöte, J. Phys. A 16, L657 (1983).

7J. L. Cardy, J. Phys. A 17, L385 (1984).

${ }^{8}$ A. M. Polyakov, Pis'ma Zh. Eksp. Teor. Fiz. 12, 538 (1970) [JETP Lett. 12, 381 (1970)].

${ }^{9}$ M. E. Fisher, in Proceedings of the 24th Nobel Symposium at Lerum, Sweden, 1973, edited by B. Lundquist and S. Lundquist (Academic, New York, 1973), p. 16.

${ }^{10}$ Conformal invariance has been used to determine the bulk critical exponents and correlation functions of twodimensional systems by A. A. Belavin, A. M. Polyakov, and A. B. Zamolodchikov, Nucl. Phys. B 241, 333 (1984); V. S. Dotsenko, ibid. 235, 54 (1984); D. Friedan, Z. Qiu, and S. Shenker, Phys. Rev. Lett. 52, 1575 (1984).

11J. L. Cardy, Nucl. Phys. B 240, 514 (1984).

${ }^{12}$ K. Binder, Z. Phys. B 43, 119 (1981).

${ }^{13}$ H. Saleur and B. Derrida, J. Phys. (Paris) 46, 1043 (1985).

${ }^{14}$ V. Privman and M. E. Fisher, Phys. Rev. B 30, 322 (1984).

${ }^{15}$ R. B. Pearson, Phys. Rep. 103, 185 (1984); M. N. Barber, R. B. Pearson, D. Toussaint, and J. L. Richardson, Phys. Rev. B 32, 1720 (1985); H. Blöte and A. Bruce (unpublished).

${ }^{16} \mathrm{E}$. Brézin and J. Zinn-Justin, Nucl. Phys. B (to be published).

${ }^{17}$ E. Eisenriegler (unpublished).

${ }^{18}$ P. Kleban, G. Akinci, R. Hentschke, and K. R. Brownstein (unpublished).

${ }^{19}$ Coordinate transformations of the small conformal group give a one-to-one mapping of the infinite $d$-dimensional space onto itself. In general dimension $d$, the small conformal group consists of homogeneous translations, rotations, and dilations and special transformations $\mathbf{r}^{\prime}\left|\mathbf{r}^{\prime}\right|^{-2}=\mathbf{r}|\mathbf{r}|^{-2}+\mathbf{a}$, where a is an arbitrary constant vector. In $d=2$ such transformations are generated by analytic functions $w(z)=(a z+b) /(c z+d)$, where $a, b, c$, and $d$ are arbitrary complex constants. The requirement that the bulk $n$-point correlation function $G_{\infty}$ satisfy Eq. (2.1) with $G_{g^{\prime}}=G_{g}=G_{\infty}$ for any transformation of the small conformal group is a stronger restriction on the spatial dependence of $G_{\infty}$ than ordinary scaling. The known four-point function for the two-dimensional Ising model, given in Eq. (2.5), is compatible with the stronger restriction. For more details, see Refs. 8 and 9.

${ }^{20}$ A. Luther and I. Peschel, Phys. Rev. B 12, 3908 (1975). Dotsenko (Ref. 10) has rederived the result with an approach based on conformal invariance.

${ }^{21}$ See, e.g., V. I. Smirnov, A Course of Higher Mathematics (Pergamon, Oxford, 1964), Vol. 3.

${ }^{22}$ L. M. Milne-Thomson, in Handbook of Mathematical Functions, edited by M. Abramowitz and I. A. Stegun (Natl. Bur. Stand., Washington, 1964), p. 567 and p. 587.

${ }^{23} \mathrm{~N}$ has, of course, the dimension of length. Taking the total magnetization $M$ to be dimensionless, one sees that $\rho$ has the dimension (length) ${ }^{-2}$ and $B$ the dimension (length) ${ }^{1 / 4}$. Since $m_{2}(N)$ and $m_{4}(N)$ have the dimension (length) ${ }^{-2}$, as follows from Eqs. (3.1), one verifies that Eqs. (3.4) and (4.4) are dimensionally consistent.

${ }^{24}$ Predictions (Ref. 11) of conformal invariance for critical behavior in wedge geometries have been confirmed by J. L. Cardy and S. Redner, J. Phys. A 17, L933 (1984) and by M. N. Barber, I. Peschel, and P. A. Pearce, J. Stat. Phys. 37, 497 (1984).

${ }^{25}$ Predictions (Ref. 7) of conformal invariance for the amplitude $A_{\xi}$ of the correlation length in strips with free boundary conditions are confirmed in T. W. Burkhardt and I. Guim, J. Phys. A 18, L25 (1985); 18, L33 (1985); and G. v. Gehlen, V. Rittenberg, and $\mathbf{H}$. Ruegg (unpublished).

${ }^{26}$ Suppose that the integral $\langle f\rangle=\int_{0}^{1} d x f(x)$ is approximated by $f_{\mathscr{N}}=\mathscr{N}^{-1} \Sigma_{i} f\left(x_{i}\right)$ with the $x_{i}$ chosen randomly in the interval $0<x_{i}<1$. Since

$$
\left\langle\left(f_{\mathscr{N}}\right)-\langle f\rangle^{2}\right\rangle=\mathscr{N}^{-1} \int_{0}^{1} d x[f(x)-\langle f\rangle]^{2},
$$

the variance of $f_{\mathscr{N}}$ decreases as $\mathscr{N}^{-1}$ as long as $\int_{0}^{1} d x f(x)$ and $\int_{0}^{1} d x f(x)^{2}$ exist, even if $f(x)$ is singular. 